\title{
Anatomía micrográfica del folíolo de la palma neotropical Bactris gasipaes (Arecaceae)
}

\author{
Francisco Paulo Chaimsohn ${ }^{1}$, Mayra Montiel$^{2}$, Enrique Villalobos ${ }^{3} \&$ Jorge Mora Urpí $^{4}$ \\ 1. Instituto Agronómico do Paraná, Caixa Postal 129, Ponta Grossa, PR, Brasil, CEP 84.001-960. Fax: (55) 4232292829; \\ fpchaimsohn@yahoo.com.br \\ 2. Escuela de Zootecnia, Facultad de Agronomía, Universidad de Costa Rica, San José, Costa Rica; \\ mmontiel@cariari.ucr.ac.cr \\ 3. Centro de Investigación en Granos y Semillas, Universidad de Costa Rica, San José, Costa Rica. Fax: (506) 2074343; \\ villalobos.enrique@gmail.com \\ 4. Escuela de Biología, Universidad de Costa Rica, San José, Costa Rica; pejibaye@cariari.ucr.ac.cr
}

Recibido 12-V-2007. Corregido 11-V-2007. Aceptado 15-IV-2008.

\begin{abstract}
Leaf micrografic anatomy of the Neotropical palm Bactris gasipaes (Arecaceae). The economic importance of the palm Bactris gasipaes is growing in the Neotropoical region. We collected leaflets from plants under a chemical fertilization regime and a population of 5000 plants per hectare, in Costa Rica. The variety, Diamantes 10, has an ascendency fom the upper Amazon basin. We used Harries hematoxiline, eocine and standard light microscopy techniques. The presence of raphids and buliform cells was confirmed for the abaxial surface of the leaflets and for the hypodermic tissue on both sides. The absence of the Krantz anatomy was confirmed in consistence with former observations about the $\mathrm{C} 3$ photosynthesis in other species of Palmaceae. The average stomatal density on the abaxial surface was $96.87 \pm 16.31$ stomata. $\mathrm{mm}^{-2}$ and $14.20 \pm 4.05$ in the adaxial surface. Rev. Biol. Trop. 56 (2): 951-959. Epub 2008 June 30.
\end{abstract}

Key words: Bactris gasipaes, leaf micrographic anatomy, stomata frequency, Krantz anatomy.

El pejibaye (Bactris gasipaes) es una palmera con importancia económica creciente en el neotrópico. En el trópico y el subtrópico americano se cultiva para la producción de palmito (brotes vegetativos tiernos) principalmente, aunque su domesticación estuvo asociada con el aprovechamiento del fruto, cuyo consumo se remonta a épocas precolombinas. Su reciente explotación para la producción de palmito contribuye a reducir la extracción de palmas del género Euterpe del bosque Atlántico y Amazónico, actualmente en peligro de extinción.

$B$. gasipaes es originario del trópico húmedo, donde la precipitación anual es igual o superior a los $2500 \mathrm{~mm}$, con períodos secos no mayores de cuatro meses. Se le encuentra en regiones con promedios anuales de temperatura iguales o superiores a $24{ }^{\circ} \mathrm{C}$. En su hábitat original, la temperatura es inferior a este valor y la humedad relativa es muy alta, debido al sombreo producido por el bosque natural (Mora Urpí y Gainza 1999).

La hoja de la palma es el principal órgano fotosintético y, por lo tanto, el responsable de la producción de biomasa, y su conocimiento es fundamental para la comprensión de varios aspectos genéticos y agronómicos (Clement y Mora Urpí 1983). Asimismo, la capacidad fotosintética intrínseca de las palmas depende de las características de la estructura foliar, como el grosor, el tamaño y el arreglo de las células del mesofilo, que determina la cantidad de tejido fotosintético por unidad de área foliar (Awal et al. 2004). El estudio de la anatomía microscópica contribuye al conocimiento y comprensión de aquellos aspectos fisiológicos 
de fundamental importancia, como los procesos de fotoasimilación, absorción, transporte, almacenamiento, e incluso, de la resistencia al ataque de plagas de insectos y enfermedades (Tomlinson 1990).

Los estomas tienen una función esencial en el mantenimiento de la homeostasis de la planta, especialmente de la regulación de la pérdida de vapor de agua y el ingreso de $\mathrm{CO}_{2}$, (Flores-Vindas 1999). Normalmente, las palmas presentan una densidad estomática que oscila entre 150 a 180 estomas.mm ${ }^{-2}$ (Larcher 1995). La frecuencia o densidad estomática (número de estomas por unidad de área), varía con las condiciones ambientales, por lo que puede diferir entre plantas de la misma especie, entre hojas de la misma planta y entre sectores de una misma hoja (Esau 1972, Brownlee 2001).

La incidencia de algunas enfermedades también puede influir en la densidad estomática por causar alteraciones en el equilibrio hídrico de la planta (Tucci et al. 2000), e interferir en la expansión de la lámina foliar. Mathew (1981) observó en Cocos nucifera una mayor frecuencia estomática en hojas de plantas enfermas $\left(228 . \mathrm{mm}^{-2}\right)$ comparado con la frecuencia estomática de hojas de plantas sanas $\left(182 \mathrm{~mm}^{-2}\right)$. Usualmente, las láminas foliares de la parte superior del dosel muestran una menor densidad estomática que aquellas de la parte superior, debido a un efecto de "dilución" por la expansión foliar (Tichá 1982).

La información sobre las características y la frecuencia de estomas en esta especie es escasa (Tucci et al. 2000, Bovi et al. 2002). Tampoco existe información de la anatomía micrográfica de los foliolos de pejibaye y eventualmente se hace referencia a esta especie, igual que a Elaeis guineensis, como especies $\mathrm{C}_{4}$, por su alta productividad. El presente trabajo es básicamente descriptivo y con él se pretendió aportar evidencia mediante microscopía de alta resolución de la ausencia de la anatomía Krantz en Bactris gasipaes, aspecto que no se encuentra documentado en la literatura.

El objetivo de este trabajo fue determinar si esta planta es $\mathrm{C}_{3}$ ó $\mathrm{C}_{4}$, así como describir la anatomía de los foliolos.

\section{MATERIALES Y MÉTODOS}

Estudio micro anatómico: Los folíolos para el estudio micro anatómico fueron obtenidos de plantas jóvenes cultivadas para palmito de la variedad Diamantes 10 de ascendencia del alto Amazonas, en la Estación Experimental. Diamantes, ubicada en Guápiles, Costa Rica ( $10^{\circ} 22^{\prime} \mathrm{N}$ y $83^{\circ} 76^{\prime} \mathrm{W}$, altitud de $\left.249 \mathrm{~m}\right)$. Después de extraídos, los foliolos se mantuvieron en bolsas plásticas con papel absorbente húmedo hasta iniciar su procesamiento, aproximadamente $3 \mathrm{~h}$ después.

El material se fijó en una solución de glutaraldehído $(2,5 \%)$ y paraformaldehído al $2 \%$ en un amortiguador de fosfato de sodio $(0,1 \mathrm{M}$, $\mathrm{pH} 7,4)$ por 24 horas. Luego, el material fue procesado mediante la siguiente metodología: a. el proceso de preparación del material fue automatizado y hecho en máquina deshidratadora VIP; b. la inclusión se realizó en un molde de metal; c. después de enfriado fue cortado en un micrótono de 6 micras; d. los cortes se pusieron en xilol y alcohol (2 min.); e. se lavó por 30 seg. y se dejó en hematoxilina de Harries por 5 min.;f. se lavó y se decoloró con alcohol ácido; g. se lavó y se pasó por el virador $(\mathrm{NaOH} 3 \%$ en agua) y por agua amoniacal; h. después de lavado por sumergimiento se aplicó eocina por 2 min; se lavó y se pasó por una secuencia de alcohol 95 y $100{ }^{\circ}$ G.L. para deshidratar; j. finalmente se montó el material en las láminas.

Las láminas fueron observadas y fotografiadas en un microscopio óptico Nikon modelo SKe, con cámara fotográfica NFX-35 y sistema fotográfico AFX-DX $\mathrm{II}_{\mathrm{II}}$, usando aumentos de 100 y 200 veces $(20$ y 40 x de aumento del microscopio, respectivamente, por $5 \mathrm{x}$ de aumento del lente de la cámara).

Frecuencia de estomas: Se obtuvieron muestras de plantas con 22.5 meses de edad provenientes de una parcela experimental con una densidad de población de 5000 plantas por hectárea, a la que se le aplicó fosfato diamónico a los 40 días de la siembra y en el cuarto, quinto, sexto y séptimo mes después de la siembra, 
para promover el desarrollo de las raíces de las plantas y homogeneizar la plantación experimental. A partir del octavo mes se aplicó, trimestralmente, abono de la fórmula comercial 18-5-15-6-0,7 siguiendo las recomendaciones para la producción comercial de esta especie en Costa Rica (Molina 2000). Para el estudio microscópico se seleccionaron diez folíolos (cinco de cada lado) de la parte central de las hojas 1 a 6 (1: hoja más joven abierta; 6 : hoja más madura). La evaluación de la densidad de estomas se hizo en foliolos obtenidos de la tercera hoja; para ello se aplicó una película de esmalte transparente para uñas que una vez con los estomas impresos se montó en un portaobjetos. Para cuantificar los estomas se utilizó un microscopio óptico (Nikon 6 x 6 Stage) acoplado a una pantalla Sony (HR Trinitron) con un campo visual equivalente a $1 \mathrm{~mm}^{2}$, con un aumento de $200 \mathrm{X}$.

\section{RESULTADOS}

Micro anatomía foliar: como en los folíolos de la mayoría de las palmas con hojas pinadas (Tomlinson 1990), los de B. gasipaes poseen una nervadura central prominente (Fig. 1A) en la superficial abaxial, característica de hojas no duplicadas, la cual presenta: epidermis (e), constituida por una capa de células angostas y rectangulares; hipodermis (h): también constituida por solo una capa de células alargadas, pero mayores que las células de la epidermis y sin cloroplastos; el clorénquima (cl): que es la capa que sigue a la hipodermis, constituida por células teñidas de rojo por la hematoxilina, redondeadas, de diversos tamaños y con paredes gruesas.

Hacia la parte interna del clorénquima, se observa un anillo de esclerénquima (ec), tejido constituido por varias células de pared secundaria, las cuales son redondeadas, con paredes engrosadas y lignificadas. En el interior del corte transversal de la nervadura se observan varios haces vasculares (hv), cuyos elementos principales son los vasos de metaxilema $(\mathbf{m x})$, vasos de protoxilema (px) y pequeñas acumulaciones de floema (f). Entre los haces se encuentra el tejido parenquimatoso central (pa), que es incoloro.

La Fig. 1B muestra el detalle de un corte transversal de una nervadura central de un folíolo. Externamente está la epidermis (e), constituida de una sola capa de células de grosor. Hacia el interior se observa la hipodermis (h), la cual también está constituida de una sola capa de células de grosor, pero estas son más grandes que las epidérmicas. Debajo de este tejido, se observa un tejido clorenquimatoso desordenado (cl) y, en seguida, el tejido esclerenquimatoso (ec), constituido por gran cantidad de células más pequeñas y de paredes gruesas, que rodea el haz vascular. En el haz vascular se observan los vasos de protoxilema (px), y metaxilema (mx), además de unas pocas células floemáticas (f).

En la Fig. 1C se presenta un corte transversal del folíolo, en el cual se observa la distribución de haces vasculares de las nervaduras laterales, los cuales son muy pequeños y rodeados por tejido esclerenquimatoso. Alternando con los haces vasculares se presentan acumulaciones de tejido esclerenquimatoso (estructuras redondas). De afuera hacia adentro del corte se observa la epidermis adaxial (e) y la epidermis abaxial (e), las cuales están constituidas por una capa de células angostas y rectangulares. Hacia adentro de la epidermis, se observa una capa de células también rectangulares, pero más grandes y sin cloroplastos, que constituye la hipodermis (h).

El mesofilo está constituido por células del clorénquima (cl), las cuales son de diversas formas y tamaños. Hacia el centro de la figura se observa una acumulación de células esclerenquimatosas (ec), de forma redondeada y que proporciona resistencia al foliolo frente a fuerzas físicas (tensión, peso, presión). El haz vascular (hv) está rodeado por una capa de esclerénquima (ec), y constituido por pequeños vasos de protoxilema (px) y células floemáticas (f). En la superficie abaxial se observa un estoma (es).

En la sección terminal de un folíolo (Fig. 1D), se observa la presencia de rafidios (ra), que son cristales aciculares de oxalato de 

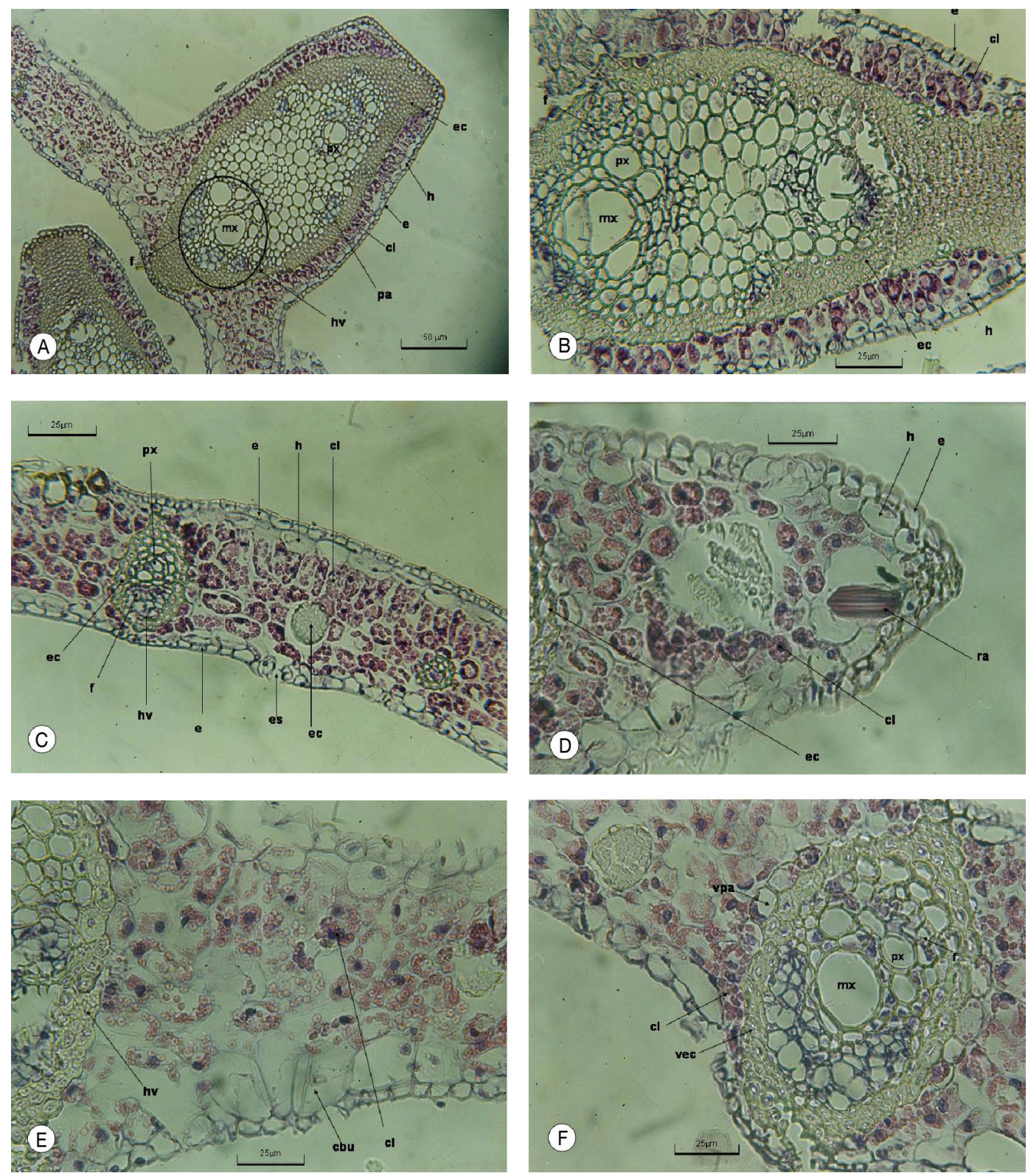

Fig. 1. Cortes transversales de segmentos de folíolos de B. gasipaes: A y B. nervadura central. C. lámina. D. Sección terminal con rafídios. E. Lámina con la presencia de células buliformes. F. Haz vascular, cuya estructura evidencia el tipo de fotosíntesis $\mathrm{C}_{3}$.

e, epidermis; h, hipodermis; cl, clorénquima o células clorenquimatosas; ec, esclerénquima o células esclerenquimatosas; hv, haces vasculares; mx, metaxilema; px, protoxilema; f, floema o células floemáticas; pa, parénquima; es, estomas; ra, rafidios; cbu, células buliformes; vec, vaina de esclerénquima; vpa, vaina de parénquima. 
calcio, en una célula parenquimatosa. Otras estructuras observadas son: la epidermis (e), la hipodermis (h), células clorenquimatosas (cl) y células esclerenquimatosas (ec).

En la superficie abaxial del folíolo (Fig. 1E) se observan las células buliformes (cbu), las cuales son células más alargadas, que acumulan agua y están involucradas en la turgencia, el despliegue o enrollamiento de los folíolos. También se observan células del clorénquima (cl) con acumulaciones de cloroplastos y parte de un haz vascular (hv) con sus bandas esclerenquimatosas.

La Fig. 1F muestra la evidencia del tipo de la fotosíntesis $\mathrm{C}_{3}$ en Bactris gasipaes, porque se observa un haz vascular que solo presenta una vaina de parénquima rodeando el haz. Internamente se observa un gran vaso de metaxilema (mx), diversos vasos de protoxilema (px), y tejido floemático desordenado (f). Rodeando el haz, se observa inicialmente una vaina de esclerénquima (ec) de paredes muy gruesas y una vaina de parénquima (vpa), como ya se mencionó. Alrededor, se observan células del clorénquima con acumulaciones de cloroplastos (cl).
Frecuencia de estomas: El pejibaye es una especie anfiestomática. La frecuencia promedio de estomas en la superficie abaxial fue de $96.87 \pm 16.31$ estomas $\mathrm{mm}^{-2}$. La frecuencia promedio de estomas en la superficie adaxial fue de $14.20 \pm 4.05$ estomas $\mathrm{mm}^{-2}$. No hubo diferencia en la frecuencia de estomas de la superficie adaxial en función de la edad de la hoja (Fig. 2), mientras que la frecuencia de estomas en la superficie abaxial mostró una reducción con la edad de la hoja.

\section{DISCUSIÓN}

La epidermis de los foliolos del pejibaye es similar al de palmas del género Sabal y Syagrus (Pérez y Rebollar 2003, Leite y Scatena 2001). Asimismo, Tomlinson (1990) señala que el tejido periférico clorenquimatoso, continuo con el tejido de asimilación de la lámina está siempre presente en las palmas, lo cual se verificó en $B$. gasipaes. De la misma forma, el tejido parenquimatoso central, que es incoloro, está presente en $B$. gasipaes como en todas las palmas, a excepción de aquellas de los géneros Phoenix, Lepidocaryum, Mauritiella y Mauritia.

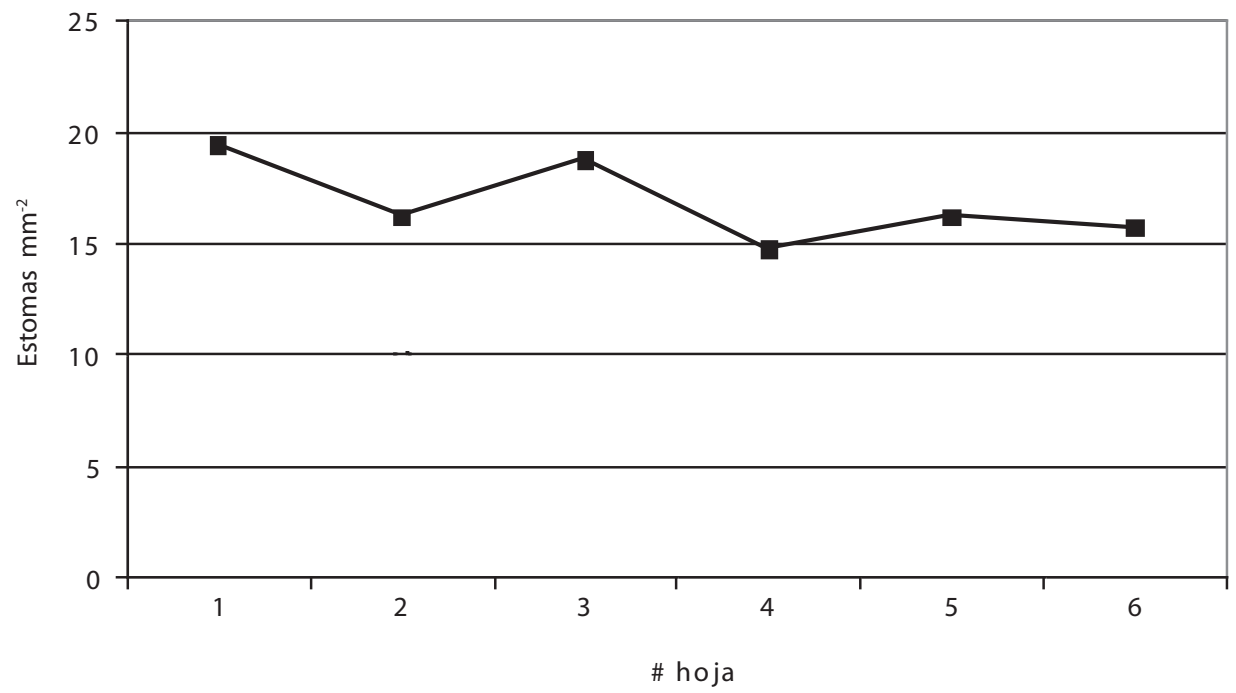

Fig. 2. Número de estomas por $\mathrm{mm}^{2}$ en la superficie adaxial en función de la edad de la hoja. 
La hipodermis se observó en todas las secciones del foliolo del pejibaye; este tejido está frecuentemente ausente en palmas que se desarrollan bajo una sombra densa, pero está muy bien desarrollado en aquellas especies de zonas áridas (Tomlinson 1990). En casos excepcionales, como en Asterogyne martiana y A. spicata esta presente una hipodermis solamente en la superficie abaxial (Stauffer et al. 2003).

En los cortes transversales de segmentos de foliolos de Bactris cubensis, B. plumeriana y $B$. jamaicana, presentados por Salzman y Judd (1995), se observan similitudes con el segmento presentado en la Fig. 1C, principalmente de la especie $B$. jamaicana. Sin embargo, $B$. cubensis presenta muchos fajos fibrosos no vasculares localizados debajo de la hipodermis adaxial, los cuales no fueron observados en $B$. gasipaes.

El oxalato de calcio, presente en los folíolos de pejibaye en la forma de rafidios (Fig. 1D) es la sal de calcio más frecuente y se encuentra en la mayoría de las familias vegetales (Esau 1972). Además de los rafidios, las monocotiledóneas pueden tener cristales de oxalato de calcio del tipo estilóideo y drusa (Prychid y Rudall 1999). Se encuentran células con cristales de oxalato de calcio en todas las partes de las palmas (Tomlinson 1990, Zona 2004). Aunque Tomlinson (1990) menciona que frecuentemente las raíces no las poseen, Zona (2004) afirma que ocasionalmente también son encontrados en estos órganos en palmas. El mismo autor observó rafidios en embriones de palmas de las subfamilias Areceae y Caryoteae; pero fueron raros en embriones de Coryphoideae y Ceroxyloideae y ausentes en Bactris major. Pérez y Rebollar (2003) encontraran gran cantidad de cristales del tipo drusa en células foliares de Sabal mauritiiformis, S. mexicana y $S$. yapa. Las células buliformes, observadas en la superficie abaxial del folíolo, son frecuentes en todos los órdenes de monocotiledóneas, excepto en las helobiales (Alismatidae) (Esau 1972).

La evidencia microanatómica permite descartar la presencia de la anatomía Krantz en Bactris gasipaes de forma clara, lo cual es prueba de la inexistencia del metabolismo $\mathrm{C}_{4}$ en esta especie. Este hallazgo no resulta sorprendente de manera alguna y tiene, más bien, carácter confirmatorio al asociarlo con lo que se conoce del mecanismo fotosintético en la familia Palmaceae y con su origen del bosque tropical húmedo de América (Mora Urpí y Gainza 1999), donde las condiciones climáticas no constituyen el hábitat de origen típico de las plantas $\mathrm{C}_{4}$. No obstante, resulta de interés presentar esta evidencia, en tanto que no existe información microanatómica al respecto y esporádicamente, por su alta productividad, se hace referencia a esta especie como representante de las especies $\mathrm{C}_{4}$, como también suele ocurrir con la palma aceitera. Es bien conocido que las plantas $\mathrm{C}_{4}$ presentan mayor adaptación en aquellas regiones con clima árido y/o con altas temperaturas y difícilmente se les encuentra en ambientes de sombra o fríos (Shibles y Villalobos 2001; Percy y Ehleringer 1984).

Según Larcher (1995) las palmas presentan una tasa de asimilación de 9,5 a $16 \mu \mathrm{mol} . \mathrm{m}^{-2} \cdot \mathrm{s}^{-1}$ de $\mathrm{CO}_{2}$. Oliveira et al (2002) observaron, una asimilación promedio de $\mathrm{CO}_{2}$ de $10,1 \mu \mathrm{mol} . \mathrm{m}^{-}$ ${ }^{2} . \mathrm{s}^{-1}$, en plantas de pejibaye con 12 meses de edad y bajo irrigación.

Las palmas presentan, generalmente, estomas localizados solamente en la superficie abaxial o, cuando ocurren en ambas superficies, son más numerosos en la superficie abaxial (Tomlinson 1990). Esta distribución diferencial de los estomas en ambas superficies foliares es muy común en las especies cultivadas (Tichá 1982). El pejibaye es una especie anfiestomática, como mencionado por Tucci et al. (2000). La frecuencia promedio de estomas en la superficie abaxial fue similar al observado por estos autores $\left(101.35 . \mathrm{mm}^{-2}\right)$, cuyas plantas crecieron en condiciones climáticas distintas al del presente trabajo (temperatura promedio anual de $22.4{ }^{\circ} \mathrm{C}$, temperatura máxima absoluta promedio de $37.6{ }^{\circ} \mathrm{C}$, precipitación anual de 1430 mm y HR promedio de 47.6 a $72.2 \%$ ) (FEGRI/ UNICAMP, 2005). Otros autores (Bovi et al. 2002) han informado de una frecuencia estomática en la superficie abaxial mucho menores a las que se observaron en esta investigación, 
en plantas cultivadas para palmito en Ubatuba, Brasil, en condiciones tropicales de alta temperatura y humedad, similares a las que prevalecieron en Guápiles, durante la ejecución de este trabajo. Tucci et al. (2000) y Bovi et al. (2002) también encontraron una frecuencia estomática, en la superficie adaxial, inferior (7,16 y 6,10 estomas. $\mathrm{mm}^{-2}$ respectivamente) a los valores nuestros. Evidentemente, la frecuencia estomática resulta altamente variable con las condiciones de clima y las prácticas culturales, no solamente en pejibaye, sino en otras palmas como la palma aceitera, donde se han encontrado variaciones cercanas al $90 \%$ en la frecuencia de estomas en la superficie abaxial de los foliolos (Zobel y Liu 1980).

Dichos autores también mencionan la densidad estomática para otras especies de palma, cuyo primer valor se refiere a la superficie abaxial: Caryota mitis (50 y 1 estomas. $\mathrm{mm}^{-2}$ ), Dictyosperma alba $\left(140 \mathrm{y}<1\right.$ estomas. $\left.\mathrm{mm}^{-2}\right)$, Plychosperma macarthuri $(110 \mathrm{y}<1$ estomas. $\mathrm{mm}^{-2}$ ), Sabal umbraculifera (310 y 270 estomas. $\mathrm{mm}^{-2}$ ) y Washington filifera (130 y 120 estomas. $\mathrm{mm}^{-2}$ ). Las dos últimas especies, que presentan una frecuencia estomática adaxial relativamente alta, son de un altiplano y de un oasis del desierto, respectivamente. Además de los géneros Sabal y Washington, los estomas son igualmente numerosos en ambas superficies en palmas de los géneros Butia, Phoenix, Corypha y otras coryphoides (Tomlinson 1990). En hojas de la palma Astrocaryum jauari, creciendo en un área periódicamente inundada, se constató la presencia de estomas solamente en la superficie abaxial (Schlüter et al. 1993).

Asimismo, Bongers y Popma (1990) determinaron la frecuencia de estomas en Bactris trichophylla (82 estomas. $\left.\mathrm{mm}^{-2}\right)$, además de otras tres especies de palma (Astrocaryum mexicanum, Chaedorea schiedana, y Chamaedorea tepejilote), cuya densidad estomática, en la superficie abaxial, fue de 105, 78 y 188 estomas. $\mathrm{mm}^{-2}$, respectivamente. En palmas de diferentes especies del género Syagrus $(S$. coronata, S. flexuosa, S. harleyi, S. microphylla, $S$. vagans y $S$. werdermannii), encontradas la Chapada Diamantina (Bahia, Brasil), donde la precipitación anual varía entre 500 a 1000 $\mathrm{mm}$, Leite y Scatena (2001) determinaron una frecuencia estomática abaxial variando entre 149 a 480 estomas.mm-2. Pereira Netto et al. (1999) mencionan que la presencia de estomas en las dos superficies de la hoja es considerada un mecanismo adaptativo para maximizar la conducción del $\mathrm{CO}_{2}$, cuando la luz y el agua no son factores limitantes. Los autores señalan que la baja frecuencia estomática, en la superficie adaxial, favorece la eficiencia en el uso del agua mediante la reducción de la pérdida de agua, cuando una alta radiación solar incide en tal superficie y por la disminución de la concentración interna de $\mathrm{CO}_{2}$ de la hoja.

Las diferencias en la densidad estomática en función de la edad de la hoja se asocian a un efecto de "dilución", debido a la expansión de la lámina foliar en respuesta al autosombreo que al abarcar un número de estomas definido genéticamente a partir de las células madre de los estomas, origina ese efecto. También podría darse un efecto de discontinuidad en la formación de los estomas en respuesta a a diferentes factores ambientales (v.gr. déficit hídrico) que haría disminuir la densidad estomática en hojas viejas (Tichá 1982). Sin embargo, el autosombreo, que podría contribuir a una mayor diferencia en la frecuencia estomática en hojas de diferentes edades, parece no ser tan acentuado en plantas de pejibaye cultivado para palmito, que está sujeto a una poda sistemática de los tallos de mayor altura. No sucede lo mismo en la palma de aceite (Zobel y Liu 1980) donde la arquitectura misma de la planta favorece el autosombreo.

\section{RESUMEN}

Se estudiaron hojas de plantas jóvenes cultivadas para producción de palmito de la variedad Diamantes 10 de ascendencia del Alto Amazonas. Se estudió la anatomía micrográfica foliar y la frecuencia de estomas en la superficie adaxial y abaxial de los foliolos de Bactris gasipaes provenientes de una plantación experimental en un régimen de fertilización química y una densidad de población de 5000 plantas por hectárea, en Guápiles, Costa Rica. Se confirmó la presencia de rafidios y células buliformes en la superficie abaxial de las láminas foliares y del tejido hipodermico en ambos lados. Confirmamos la ausencia 
de la anatomía Krantz en esta especie, reafirmando la fotosíntesis $\mathrm{C}_{3}$ como mecanismo metabólico en la familia Palmaceae. La densidad promedio de estomas en la superficie abaxial fue de $96,87 \pm 16,31$ estomas. $\mathrm{mm}^{-2}$ y en la superficie adaxial fue de $14,20 \pm 4,05$ estomas. $\mathrm{mm}^{-2}$.

Palabras clave: Bactris gasipaes, anatomía micrográfica, frecuencia de estomas, anatomía de Krantz.

\section{AGRADECIMIENTOS}

Agradecemos al CNPq-Brasil, por el otorgamiento de la beca de doctorado al primer autor, sin la cual esto trabajo no se hubiera realizado. Al Centro de Investigación en Granos y Semillas de la Universidad de Costa Rica por la indispensable ayuda financiera y logística. A María del Carmen Obando por su fundamental apoyo en el montaje del tejido foliar. A Guillermo Vargas por su colaboración en el material fotográfico. A Guillermo Solano por el imprescindible apoyo en el trabajo de campo.

\section{REFERENCIAS}

Awal, M.A., W. Ishak, J. Endan \& M. Haniff. 2004. Determination of specific leaf area and leaf area-leaf mass relationship in oil palm plantation. Asian J. Plant Sci. 3: 264-268.

Bongers, F. \& J. Popma. 1990. Leaf characteristics of the tropical rain forest flora of los Tuxtlas, Mexico. Bot. Ga. 151: 354-365.

Bovi, M.L.A., G. Godoy Jr, M.L.S. Tucci \& S.H. Spiering. 2002. Nitrogen deficiency in peach palm (Bactris gasipaes Kunth). An. del XXVI Int. Hort. Congress. ISHS, Toronto, Canadá.

Brownlee, C. 2001. The long and the short of stomatal density signals. Trends Plant Sci. 6: 441- 442 .

Clement, C.R. \& J. Mora-Urpí. 1983. Leaf morphology of the pejibaye palm (Bactris gasipaes H.B.K.). Rev. Biol. Trop. 31: 103-112.

Esau, K. 1972. Anatomía vegetal. Omega, Barcelona, España.

Flores-Vindas, E. 1999. La planta: estructura y función. v. II. Libro Universitario Regional, Cartago, Costa Rica.
Larcher, W. 1995. Physiological plant ecology. 3 ed. Springer, Nueva York, EEUU.

Leite, K.R.B. Y V.L. Scatena. 2001. Anatomia do segmento foliar de espécies de Syagrus Mart. (Arecaceae) da chapada diamantina, Bahia, Brasil. Sitientibus Sér. Ciên. Biol. 1: 3-14.

Mathew, C. 1981. Water relations of coconut palm affected by root (wilt) disease. J. Plantation Crops 9: 51-55.

Mora Urpí, J. \& J. Gainza E., 1999. Palmito de pejibaye (Bactris gasipaes Kunth): su cultivo e industrialización. Editorial de la Universidad de Costa Rica, San José, Costa Rica.

Oliveira, M.A.J. de M.L.A. Bovi, E.C. Machado, M.M.A. Gomes \& G. Habermann, J.D. Rodrigues. 2002. Fotossíntese, condutância estomática e transpiração em pupunheira sob deficiência hídrica. Sci. Agr. 59: 59-63.

Percy, R.W. \& J. Ehleringer, 1984. Comparative ecophysiology of $\mathrm{C}_{3}$ and $\mathrm{C}_{4}$ plants. Plant, Cell Environ. 7: $1-13$.

Pereira Netto, A.B., A.C. Gabriele \& H.S. Pinto. 1999. Aspects of leaf anatomy of kudzu (Pueraria lobata, Leguminosae - faboideae) related to water and energy balance. Pesqui. Agropecu. Bras. 34: 1361-1365.

Pérez, M. \& S. Rebollar. 2003. Anatomía y usos de las hojas maduras de tres especies de Sabal (Arecaceae) de la Península de Yucatán, México. Rev. Biol. Trop. 51: 333-344.

Prychid, C.J. \& P.J. Rudall. 1999. Calcium oxalate crystals in monocotyledons: a review of their structure and systematics. Ann. Bot.-London 84: 725-739.

Salzman, V.T. \& W.S. Judd. 1995. A revision of the Greater Antillean species of Bactris (Bactridinae: Arecaceae). Brittonia 47: 345-371.

Schlüter, U.B., B. Furch \& C.A. Joly. 1992. Physiological and anatomical by young Astrocaryum jauari Mart. (Arecaceae) in periodically inundated biotopes of Central Amazonia. Biotropica 25: 384-396.

Shibles, R. \& E. Villalobos. 2001. Fotosíntesis, p. 13-51. In: E. Villalobos (editor). Fisiología de la producción de los cultivos tropicales. Fascículo 1. Procesos fisiológicos básicos. Editorial de la Universidad de Costa Rica, San José, Costa Rica. 
Stauffer, F.W., C.B. Asmussen, A. Henderson \& P.K., Endress. 2003. A revision of Asterogyne (Arecaceae: Arecoideae: Geonomeae). Brittonia 55: 326-356.

Tichá, I. 1982. Photosynthetic characteristics during ontogenesis of leaves. 7. stomata density and sizes. Photosynthetica 16: 375-471.

Tomlinson, P.B. 1990. The structural biology of palms. Clarendon Oxford, Oxford, Inglaterra.

Tucci, M.L.A., M.L.A. Bovi S. Machado \& S.H. Spiering, 2000. Stomatal frequency and size in leaves of pejibaye (Bactris gasipaes Kunth). Acta Hort. 516: 145-154.
Zobel, D.B. \& V.T. Liu, 1980. Leaf-conductance patterns of seven palms in a common environment. Bot. Ga. 141: 282-289.

Zona, S. 2004. Raphides in palm embryos and their systematic distribution. Ann. Bot.-London, 93: 415-421.

\section{REFERENCIA DE INTERNET}

FEAGRI/UNICAMP. 2005. Clima de Campinas. Cidade Universitária "Zeferino Vaz", Campinas, Brasil. (Consultado: 24 de agosto, 2005, http://orion.cpa. unicamp.br/portal/modules.php?name=Paginas Internas\&file $=$ climacps). 